\title{
Guidance effect of a parking guidance system in a middle-wide range area
}

\author{
T. Katori, K. Tatematsu \& T. Izumi \\ Nihon University, Japan
}

\begin{abstract}
In this paper, we describe the guidance effects of a parking guidance system by traffic simulator.

First, to compare the guidance effects of the systems, we constructed a cellautomaton-simulator. To purely evaluate the guidance effect by the simulator, we measured the total time for a round trip between origin and destination, without other traffic passing.

A driver's parking choice is based on Katori et al.'s “Comparison between automatic distribution and driver's feature in parking guidance system”, 2006. If a driver has no knowledge concerning parking availability, in other words, if there is no parking guidance system, the driver chooses a nearest parking spot from his own destination. If drivers are provided with information by information boards, they consider the parking situation (full congested or empty), and they choose a parking location. The parking situation information is renewed at each intersection where the boards stand. We define the "driver's parking choice" as a combination of information about the parking situation, destination and car park location. We compare the guidance effects under these circumstances.

The area under consideration is east Tokyo to west Chiba along the Edogawa River, and this area includes some living zones. The parking lots in this area are managed by the parking situation information system, and we show the quantitative evaluation values of the guidance effect.
\end{abstract}

Keywords: parking guidance system, guide effect, driver's park choice, cell automaton simulator, middle wide area. 


\section{Introduction}

A parking guidance system is a system to inform drivers about the actual situation (full, congested or empty) of car parks in city centres.

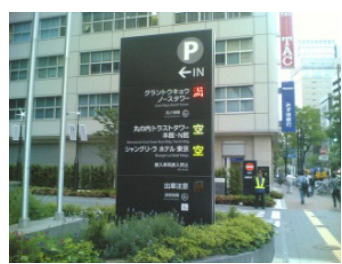

(a)

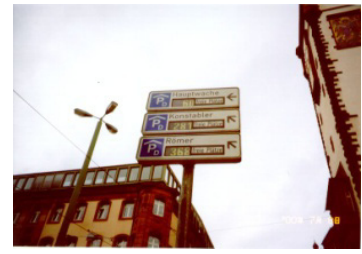

(b)

Figure 1: $\quad$ Some examples of car park information boards; (a) in Japan and (b) in Frankfurt.

The system was installed around 2000 during the ITS boom [1], but the system was installed without sufficiently considering the effect. Accordingly, in Japan nowadays, there are local areas where the system is not put to full use.

The original system shows only car park location on the information boards. Drivers can only make assumptions about the occupation level of the parking lot, before they reach the parking lot nearest to their destination.

Improving the driver's expectation by providing correct information about the car park situation will help solve the above problem, but it is still difficult in reality. However, nowadays, drivers can receive car park information at any time by car-navigation-system or their mobile phone. So, it is possible to improve the system's effectiveness by providing drivers with useful information contents for drivers, if an expectation cannot improve. Of course, all responsibility by the information belong drivers, any drivers may not follow the guides.

Against the background above, this paper describes the effect of the parking guidance system by traffic simulator. The comparison guide methods are the following three cases.

- Case One: There is no parking guidance system (Drivers drive to a car park nearest to their destination. Drivers decide the car park location at the start of their trip and do not change that choice.)

- Case Two: A parking guidance system is installed (Drivers drive to a car park that is nearby and where it is possible to park. Drivers can be provided with renewed parking situation information, and they follow the guidance information exactly)

- Case Three: A parking guidance system is installed like in Case Two, but drivers receive regular updates about which car park they should park at.

The effects of the guidance information are compared by a traffic simulator operation [3] constructed originally by the authors. This simulator adopts a cell automaton model, but does not consider other passing traffic, congestion due to 
intersection signals, and so on, because the simulator is to purely measure guidance information effect.

A driver's car park choice is based on reference [1]. If a driver has no knowledge concerning parking availability, in other words, if there is no parking guidance system, the driver chooses the nearest parking spot from his own destination. If drivers are provided with information by information boards, they can consider the car park situation (full, congested or vacant), and they can choose a parking location. On information boards at intersections, parking statements are renewed. We define the "driver's parking choice" as a combination of information about the parking situation, destination and car park location. We compare the guidance effects under these circumstances.

The main evaluation value is the average trip time. To purely evaluate the guidance effect by the simulator, we measured the total time for a round trip between origin and destination, without other traffic passing. The clear effects of the parking guidance system are shown by the simulation.

The area under consideration is east Tokyo to west Chiba along the Edogawariver, and this area includes some living zones [4]. The car parks in this area are managed by the system, and we show the quantitative evaluation values of the guidance effect.

Finally, we show that the guidance effect by information from mobile terminals is the same as a parking guidance system.

\section{Components of the parking guidance system}

In this paper, we compare the effect for some phases of a parking guidance system. In this section, we explain the different components in these phases.

\subsection{Standing parking information boards}

Figure 2 shows the components of a parking guidance system by standing boards.

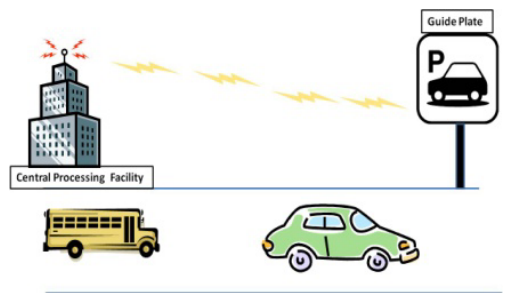

Figure 2: Composition of parking guidance system.

Information concerning the level of congestion for each car park is communicated to the management centre, and the information is shown on the boards on the road side. Drivers decide their parking location based on the information, their own destination and position. 


\subsection{Advanced parking guidance system}

Nowadays, drivers can receive parking information and location everywhere by car-navigation system or mobile terminal without the boards. Figure 3 shows the component of the advanced parking guidance system.

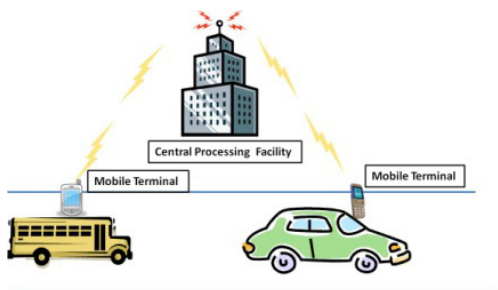

Figure 3: $\quad$ Composition of real parking guidance.

The sensor of parking information and the integration section are the same as the previous system, but drivers can receive the information directly from a beacon or a base station from a traffic information system. In this phase, the drivers can receive the information of more remote car parks and not written on the boards.

\section{Driver's car park choice model}

To measure the parking guidance effect, it is important to analyse which parking choice the driver makes depending on the provided guide information. The driver's car park location choice model is explained in this section, including also a case where no parking guidance system is available. Generally, a driver's destination is not a car park. So in this study, we also assume that the driver's final destination differs from the car park.

\subsection{Without a parking guidance system}

This case means drivers do not judge parking possibilities until they reach the car park.

Drivers decide to park nearest to their destination at their starting time. They cannot receive renewed information while they are driving, and if the car park is full at their arrival, they try to find another car park nearby.

\subsection{Standing parking guidance boards}

If there are no guidance boards at the driver's trip origin, the drivers are not aware of information by parking guidance concerning the level of congestion at car parks. Therefore, first, the driver heads to a car park nearest to his own destination. When the driver reaches an intersection where a guidance board is standing, the driver decides again on a car park place depending on the following three conditions. 
- The distance between destination and the car park. (The driver would like to park as near as possible to his destination.)

- The number of remaining vehicles at the car park. (The more vacant parking lots available, the easier to park.)

- The distance between the car park and the current location. (If the car park is too far the empty lots might be taken by the time of one's arrival.)

The car park is decided by the feature functions shown in figure 4 .

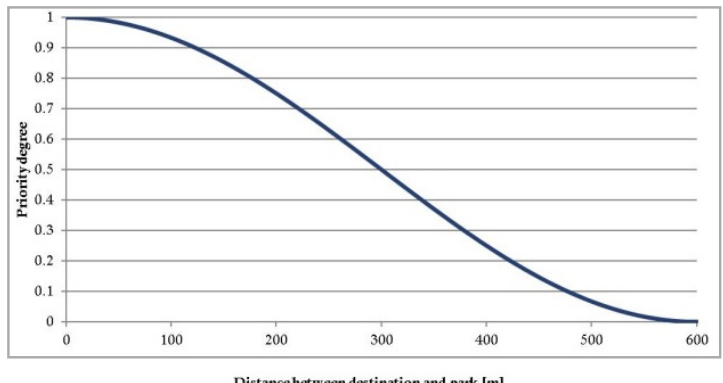

(a)

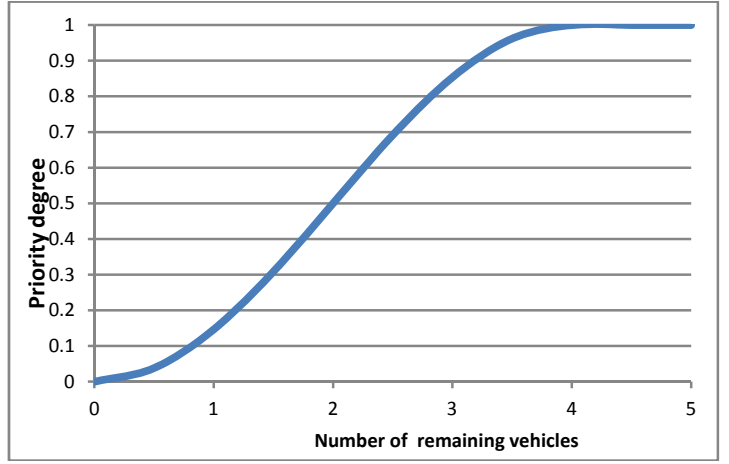

(b)

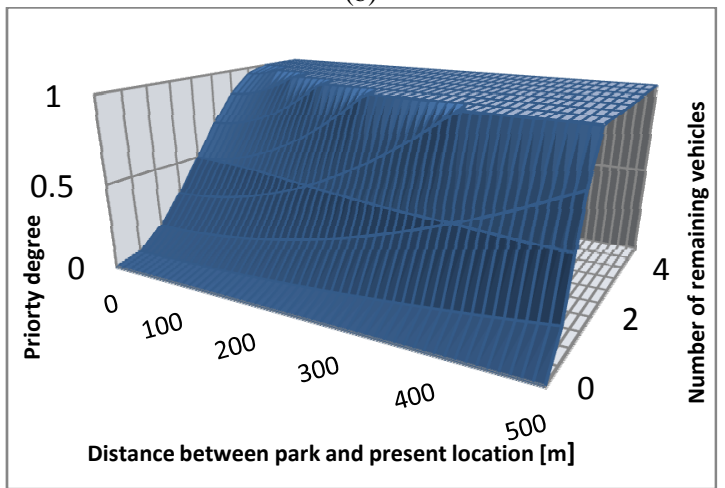

(c)

Figure 4: Feature functions to decide car park location. (a) Distance between destination and car park; (b) Number of remaining vehicles; (c) Distance between current location and car park, and the capacity. 
The distance in figure 4(a) is determined by the average distance between bus stops that a person feels possible to walk. The relation between the distance and the capacity (c) is determined by reference [1].

\subsection{Advanced parking guidance system}

This parking guidance tries to allocate the ideal distribution. Therefore, in this case it is not how the driver decides a car park based on available information. The driver's car park wish is considered, but if the driver has no clear preference between several car parks [1], the driver is treated as a wildcard. The method optimizes the whole distribution to the ideal.

Under this circumstance, the driver can receive car park information anytime and anywhere. So, the driver chooses and decides the car park at the start of the trip. At the time, there are four conditions to decide on a car park:

- The distance between the destination and the car park;

- The number of remaining vehicles at the car park;

- The distance between the car park and the current location;

- The direction/angle between the destination and the park.

The first three conditions are the same as 3.2, but the last one is an additional condition because the driver can receive all locations by GPS. If there are several car parks at the same distance from the destination, this feature is expressed on the driver's route.

$\mathrm{O}$ is the driver's origin location, $\mathrm{P}$ is the car park location and $\mathrm{D}$ is the location of the destination. If the angle OPD is obtuse, then the car park is located on the route. But if the angle is more acute, the car park is located past the destination or in a different direction. Therefore, the feature function is determined by figure 5 .

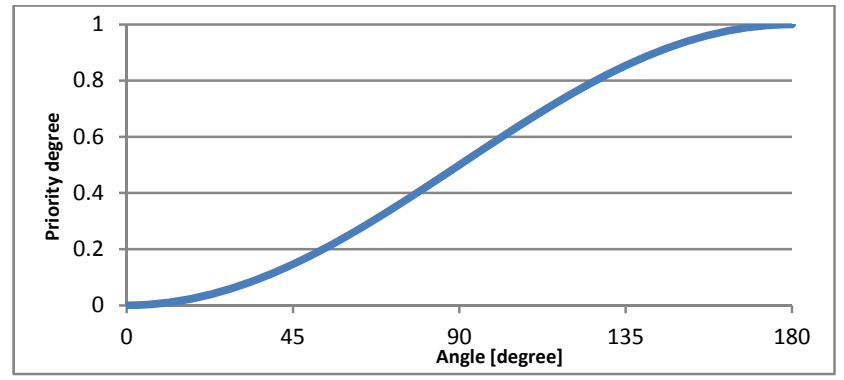

Figure 5: $\quad$ Feature function of angle between destination and car park.

Drivers can receive renewed information while driving. However, when the driver would like to change car parks, the driver can confirm the renewed information and change his route only when at an intersection, because the driver can change routes only at an intersection. 


\section{Case study}

\subsection{Area under consideration}

The area and road network under consideration is the Edogawa Ward in Eastern Tokyo to Ichikawa City in North-western Chiba along the Edogawa-river. This area spreads about $6[\mathrm{~km}]$ square and the area includes two living zones. Figure 6 shows the road network, and table 1 indicates the conditions for the network.

Table 1: $\quad$ Conditions of the area under consideration.

\begin{tabular}{|l|l|}
\hline Number of nodes (intersections and car parks) & 92 \\
\hline Number of directed links & 252 \\
\hline Number of car parks & 24 \\
\hline Capacity of all car parks & 291 \\
\hline Number of flow-in points & 4 \\
\hline Number of destinations & 4 \\
\hline Number of flow-in vehicles & 288 \\
\hline Required time at destination & $20[\mathrm{~min}]$ \\
\hline
\end{tabular}

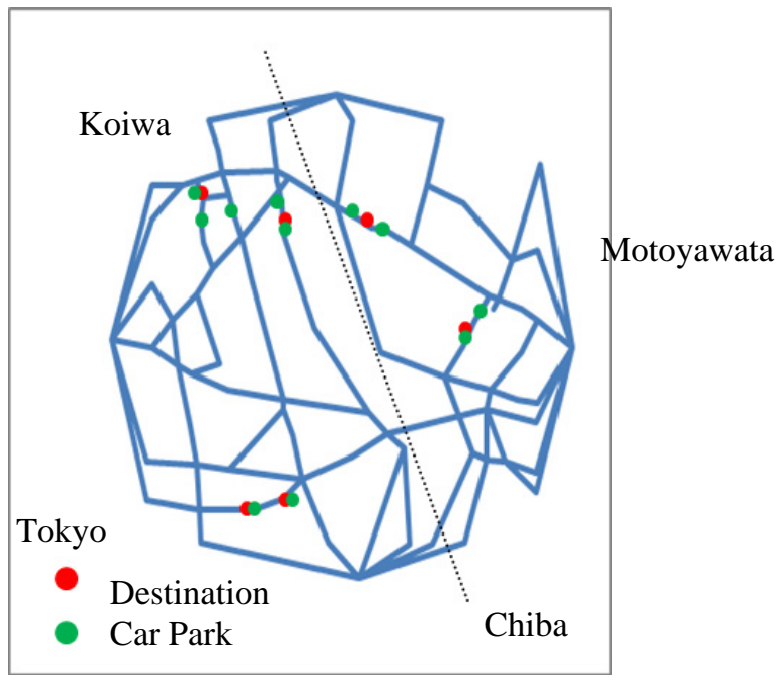

Figure 6: Road network under consideration. (East Tokyo Metropolitan Area to Northwest Chiba.)

The road network is expressed by graph data in this study, intersection and car parks are nodes, and the roads that connect intersections are directed links.

To confirm the effect of the parking guidance system, all destinations have several car parks nearby. And the numbers of flowing-in vehicles are the same as the car park capacity. Therefore, all vehicles can park somewhere. 


\subsection{Assumptions in this paper}

Assumptions in this paper are as follows:

- All car parks are managed by an integrated parking guidance system.

- All car parks are free of charge. Therefore, fee differences or road-side parking is not considered as decision elements.

- Drivers do not have a favourite car park.

- All vehicles are normal size passenger cars.

- All car park spaces are for normal size cars too. Individual parking lot access convenience is not considered.

\subsection{Cell automaton simulator}

In this section, we introduce a cell-automaton-simulator, specifically developed for this study. This simulator is purely to compare parking guidance effects. Therefore, the simulator does not consider passing traffic and traffic jams due to signals.

(1) Representation of road data

Roads are composed of cells. A cell length is $5[\mathrm{~m}]$ and the unit time is 0.5 [sec], meaning the vehicle's velocity is $36[\mathrm{~km} / \mathrm{h}]$. A vehicle goes forward when there is no vehicle in the cell ahead.

(2) Vehicle

A vehicle has the following individual elements:

- Origin;

- Departure time;

- Destination (not car park).

Flowing-in vehicles are ordered by uniformed randomized numbers. Therefore, vehicles usually flow in the same order for different guidance phases.

(3) Behaviour of vehicles in the cell-automaton-simulator

Figure 7 shows vehicle behaviour in the cell-automaton simulator.

At the starting time, an individual vehicle decides on the car park location by rules and conditions explained in 3.1-3.3 considering the location of flowing-in, destination and car parks.

The vehicle searches the shortest distance path [5] to the car park location from the current point, and flows into the cell-automaton network.

If the vehicle reaches an intersection where the vehicle can receive renewed information (from a standing guidance board or a beacon), the vehicle decides on the car park location again based on the renewed information, and changes his own route.

When the vehicle arrives at the chosen car park and can park, the vehicle parks there. The driver walks to the final destination from the car park, and the walking time is added to the trip time. At the destination, the driver accounts 20 min which means general shopping time, and afterwards, the driver walks back to the car park. 


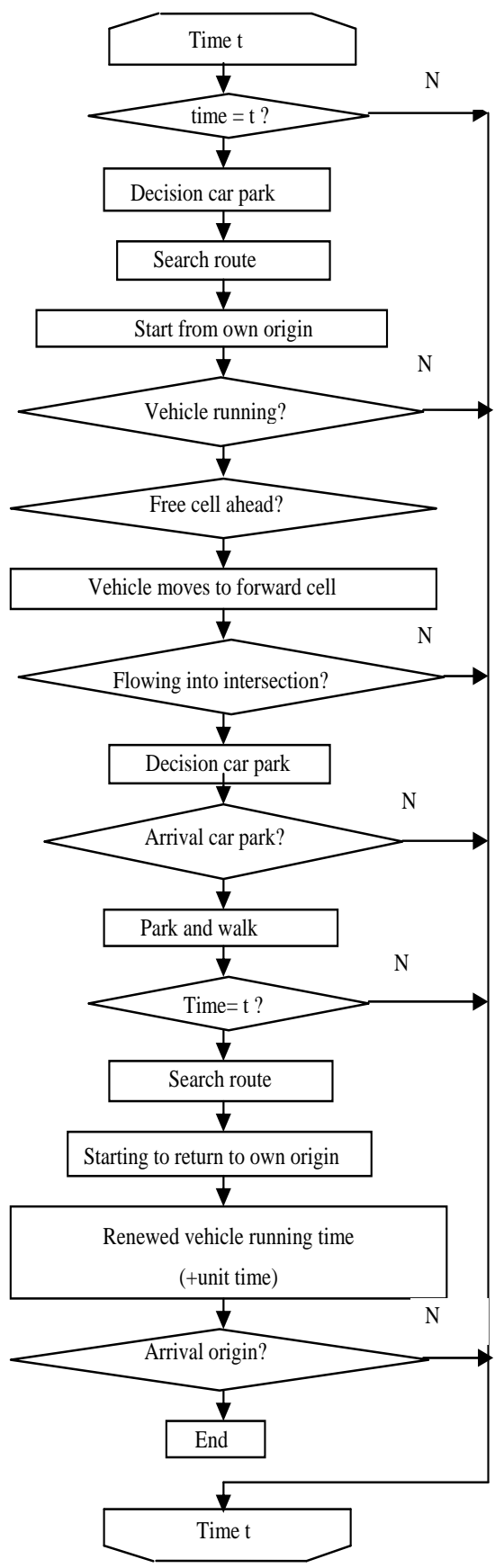

Figure 7: Flowchart of vehicle behaviour in the cell-automaton simulator. 
For the return trip, the vehicle searches the shortest path again, and leaves immediately following route.

When the vehicle comes back at a flow-in point, the trip time is stored. The time includes walking and required time at the destination.

\subsection{Evaluation value}

The evaluation value is the average trip time.

where

$$
\mathrm{E}=(1 / \mathrm{n}) \sum \mathrm{t}_{\mathrm{i}}
$$

E: evaluation value

$\mathrm{n}$ : number of flowing- in vehicle

$\mathrm{t}_{\mathrm{i}}$ : trip time of vehicle No. i (including waiting time, walking and requirement time at destination)

\subsection{Results and discussion}

Table 2 shows the average trip time for three circumstances by the simulator.

Table 2: $\quad$ Circumstances and evaluation value for parking guidance.

\begin{tabular}{|l|c|c|}
\hline \multicolumn{1}{|c|}{ Phase } & $\begin{array}{c}\text { Number of guidance boards } \\
\text { or beacons }\end{array}$ & $\begin{array}{c}\text { Average trip time } \\
{[\mathrm{min}]}\end{array}$ \\
\hline No parking guidance & 0 & 36.17 \\
\hline Parking guidance system & 86 & 32.68 \\
\hline Advanced parking guidance & 252 & 32.67 \\
\hline
\end{tabular}

The average trip time includes round-trip walking time between the car park and destination. The required time at the destination (set at 20 minutes) is also included in the average trip time.

The average trip time was reduced by the parking guidance system. The average trip time is almost the same for the parking guidance system and the advanced parking guidance system. When considering the individual trip time for 48 vehicles, there are differences between each vehicle because the vehicles can change their choice of car park at many points.

When a vehicle approaches a car park, the driver's mind changes depending on the parking availability. In other words, choosing where to park is not a oneoff decision, but might include some stray driving.

As a conclusion, the number of points to accept information and to change the parking choice should be limited in this model.

A real-time parking guidance system has the same guidance effect with or without mobile terminals. Accordingly, without investments such as the current system, a situation where the same effect can be obtained has already been realized.

As for the advanced parking guidance, the driver's car park choice feature is the angle between the car park location and the destination. Due to a small 
number of parking lots for the destination, the condition does not perform effectively in the applied network.

\section{Conclusion}

To compare the guidance effect by parking guidance systems, we constructed a cell-automaton-simulator. With the simulator, we measured the average trip time without other traffic passing. The area under consideration was the Edogawa Ward (in Eastern Tokyo) to Ichikawa City (in Western area of Chiba prefecture).

The effect of parking guides are shown by shorter average trip time, and the guide effect by parking guidance system is almost the same for the advanced guide.

Future research will have to consider, among others, the following:

- A mixed case with some vehicles following the guidance information and others not;

- Determining suitable guide points for parking guidance;

- Applying this evaluation to other areas;

- A mixed case of with some parks managed by guidance systems and others not;

- Considering if differences in parking fees lead in different results;

- Verification of cost-effectiveness for the introduction parking guidance systems.

\section{Acknowledgement}

We thank Assistant Professor Ruth Vanbaelen, Nihon University, for English language assistance.

\section{References}

[1] Teruomi Katori, Hiroshi Honda, Takashi Izumi, "Comparison between automatic distribution and driver's feature in parking guidance system”, I.E.E. 06, 4-180 (2006) (in Japanese).

[2] Fumitaka Kurauchi, Yasunori Iida, Hiroshi Tsukaguchi, Nobuhiro Uno, Makoto Kato, "The Empirical Analysis on the Evaluation of Parking Guidance and Information System”, 3rd ITS Congress (1996).

[3] Akira Fujii, Teruomi Katori, Takashi Izumi, "Guidance effect of Parking Guidance System by Parking Guidance Simulator”, I.E.E. 08, 4-212 (20083) (in Japanese).

[4] Kazuya Tatematsu, Teruomi Katori, Takashi Izumi, “To distribute vehicles by parking guidance system in middle area”, Proceedings of the 2012 IEICE General Conference, A-17-7(2012-3) (in Japanese).

[5] E. W. Dijkstra, “A note on Two Problems in Connexion with Graphs”, Numerische Mathematik 1, pp. 269-271 (1955). 